\title{
Ottimizzazione dei sistemi biologici avanzati per il trattamento delle emissioni climalteranti
}

\author{
Giuseppina Monteleone ${ }^{1}$
}

${ }^{1}$ Affiliation not available

\begin{abstract}
Le emissioni di gas climalteranti in atmosfera hanno subito un incremento nel tempo dovuto principalmente all' aumento demografico e ai processi di industrializzazione. Esse contribuiscono all'effetto serra quindi all'aumento delle temperature. $\mathrm{La} \mathrm{CO}_{2}$ è il principale gas climalterante, oggi si stima una concentrazione di circa 410 ppm ed entro la fine del secolo può superare $550 \mathrm{ppm}$ con un aumento della temperatura media globale superiore a $2{ }^{\circ} \mathrm{C}$. Bisogna intervenire con tecnologie in grado di ridurre le concentrazioni di $\mathrm{CO}_{2}$ nelle emissioni. L'attività di ricerca si è focalizzata sull'ottimizzazione di una tecnologia innovativa per la cattura di $\mathrm{CO}_{2}$ ed il simultaneo recupero di biomassa. Il sistema, brevettato dal gruppo di Ingegneria Sanitaria Ambientale (SEED) dell'Università degli Studi di Salerno, prevede l'impiego di microrganismi fotosintetici in grado di fissare $\mathrm{CO}_{2}$. È stato predisposto il funzionamento in parallelo di due fotobioreattori algali, ognuno sottoposto ad una diversa lunghezze d'onda, bianca e viola. Risultati migliori, in termini di efficienza di cattura e produzione di biomassa, si sono ottenuti con lunghezza d'onda viola.
\end{abstract}

\section{Introduzione}

L'aumento demografico e i continui processi di industrializzazione hanno richiesto una quantità sempre crescente di materie prime provocandone una forte diminuzione ${ }^{1}$. Esse vengono estratte dagli ecosistemi naturali per essere utilizzate e poi restituite sotto forma di rifiuti ${ }^{2}$. La produzione di energia, ad esempio, richiede come materia prima i combustibili fossili, i quali dopo la 
combustione rilasciano nell' atmosfera gas climalteranti, tra cui la $\mathrm{CO}_{2}{ }^{3}$. L'anidride carbonica è un gas climalterante già presente in natura ma concentrazioni elevate in atmosfera, derivanti principalmente da sorgenti di emissioni di tipo antropiche (uso dei combustibili, processi industriali, attività agricole, processi di trattamento dei rifiuti) ${ }^{4}$, contribuiscono ad aumentare le problematiche connesse all'effetto serra ${ }^{5}$. L'innalzamento delle temperature è uno dei principali problemi a livello globale in quanto provoca un aumento della superficie delle terre desertificate, un incremento del fenomeno dello scioglimento dei ghiacciai e fa aumentare la frequenza di fenomeni atmosferici estremi ${ }^{6}$. Attualmente le concentrazioni di $\mathrm{CO}_{2}$ sono pari a circa $410 \mathrm{ppm}$; e in assenza di opportune misure di controllo sono state stimate concentrazioni future fino a $550 \mathrm{ppm}$ ed oltre entro la fine del secolo, con un simultaneo aumento della temperatura media globale oltre i $2^{\circ} \mathrm{C}^{7}$. Visto che il potere climalterante della $\mathrm{CO}_{2}$ risulta essere quello più rilevante ${ }^{8}$, bisogna cercare soluzioni in grado di ridurre le concentrazioni di $\mathrm{CO}_{2}$ nelle emissioni di gas climalteranti. L'Unione Europea ha recentemente lanciato il piano "Green New Deal" con l'obiettivo di rendere "Carbon Neutral" il continente europeo entro il 2050.

Ad oggi, con lo scopo di raggiungere obiettivi di sostenibilità, sono previste tecnologie per il trattamento della $\mathrm{CO}_{2}$ classificate in CCS (Carbon Capture and Storage) e CCU (Carbon Capture and Utilization) che prevedono sequestro, compressione, trasporto e stoccaggio o utilizzo ${ }^{9}$. I metodi di sequestro della $\mathrm{CO}_{2}$ prevedono la cattura direttamente da fonti di emissione, essa può avvenire attraverso la tecnica di pre-combustone, post-combustione e ossicombustione ${ }^{10}$. Dopo la cattura e la compressione segue la fase di trasporto che avviene tramite condotte oppure su nave, dove la $\mathrm{CO}_{2}$ dev'essere compressa e liquefatta quindi risulta più costoso come metodo ${ }^{11}$. Dopo il trasporto, il destino della $\mathrm{CO}_{2}$ cambia a seconda della tecnica con cui viene trattata. Nel caso di CCS la $\mathrm{CO}_{2}$ viene immagazzinata in siti di stoccaggio aventi profondità superiori ai 1000 metri come, ad esempio, giacimenti di petrolio, gas esauriti e carbone o falde acquifere ${ }^{12}$. L'approccio CCS risulta fattibile ma con elevati consumi energetici. Il metodo CCU è una tecnologia innovativa dove la $\mathrm{CO}_{2}$ catturata viene riutilizzata per la produzione di prodotti ad alto valore aggiunto. $\mathrm{E}$ 
una tecnologia nascente ${ }^{13}$ che sta acquistando interesse tra la comunità tecnico-scientifica per le applicazioni di processi chimico-fisici e biologici. Tra le tecnologie CCU, le biotecnologie stanno attirando l'attenzione come processi sostenibili sia dal punto di vista economico che ambientale, esse si servono di microrganismi fotosintetici come le microalghe in grado di fissare la $\mathrm{CO}_{2}$ e contemporaneamente convertirla in biomassa valorizzabile ${ }^{13}$.

Le microalghe sono state scelte per l'elevata capacità fotosintetica attraverso cui sono in grado di fissare $\mathrm{CO}_{2}$ e trasformarla, attraverso reazioni chimiche, in sostanze più complesse per il nutrimento ${ }^{14}$. Per soddisfare il fabbisogno nutritivo richiedono principalmente elementi come carbonio, azoto e fosforo ${ }^{15}$. Come sottoprodotto, derivante dal processo fotosintetico, rilasciano ossigeno ma soprattutto producono biomassa ricca di carboidrati, proteine, lipidi, polimeri che può essere riutilizzata come materia prima in diversi settori ${ }^{16}$, la biomassa quindi risulta una risorsa riutilizzabile $^{17}$. I sistemi convenzionali per la coltivazione di biomassa algale possono essere aperti "Open Ponds" (Fig. 1) o chiusi "Fotobioreattori PBR" (Fig.2).

I sistemi aperti risultano economici e di facile manutenzione ma il diretto contatto con l'ambiente è un limite, perché espone la coltura a rischi di contaminazione da parte di organismi esterni ${ }^{19}$. I fotobioreattori, invece, non offrono alcuno scambio con l'ambiente esterno dato che sono formati da contenitori chiusi ${ }^{20}$. Riescono ad offrire un controllo maggiore dei parametri che ne influenzano la crescita come $\mathrm{pH}$, nutrienti, concentrazioni di $\mathrm{CO}_{2}$, intensità di luce, miscelazione, salinità ${ }^{18}$. La luce insieme alla sue caratteristiche come intensità luminosa, lunghezza d'onda, tipologia di sorgente luminosa e fotoperiodo, è un fattore di crescita influente ${ }^{21}$. Le diverse specie di microalghe sono in grado di assorbire lunghezze d'onda tra i 400 e $700 \mathrm{~nm}^{22}$. La porzione dello spettro delle radiazioni elettromagnetiche luminose è il principale fattore che determina l'incremento della crescita, oltre all'efficienza dei sistemi chiusi di coltivazione PBR microalgali ${ }^{23}$. Lo studio ha previsto un'attività sperimentale condotta presso il Laboratorio di Ingegneria Sanitaria Ambientale (SEED) dell'Università degli Studi di Salerno dove il Gruppo di ricerca di Ingegneria Sanitaria Ambientale 
ha realizzato un innovativo sistema di foto-bioreattori algali connessi a colonna di adsorbimento per la coltivazione di biomassa valorizzabile e cattura di $\mathrm{CO} 2$.

\section{Materiali e metodi}

Per il trattamento delle emissioni climalteranti è stato adoperato un sistema biologico innovativo a base di microalghe per trattare $\mathrm{la}_{2}$ contenuta nelle emissioni e per la contemporanea produzione di biomassa. La specie algale utilizzata è stata la Chlorella Vulgaris, specie eucariota di diametro variabili da 2 a $10 \mu \mathrm{m}^{24}$, coltivata in fotobioreattori con Bold's Basal Medium (BBM). Il set-up sperimentale è composto da due foto-bioreattori con funzionamento in parallelo, alimentati con stesse concentrazioni di $\mathrm{CO}_{2}$ in ingresso (5\%) e stessa portata di ricircolo $(100 \mathrm{ml} / \mathrm{min})$. I due fotobioreattori $\mathrm{PBR}_{1}$ e $\mathrm{PBR}_{2}$ differiscono per il sistema di illuminazione, in particolare per la lunghezza d'onda emessa dalle sorgenti luminose a LED che risultano essere rispettivamente bianca e viola. Scopo dell'attività è stato quello di individuare la lunghezza d'onda più efficiente tra bianca e viola in grado di massimizzare la produzione di biomassa e l'efficienza di rimozione di $\mathrm{CO}_{2}$.

Per ogni fotobioreattore sono stati disposti 4 luci LED con stessa intensità luminosa pari a 8000 lux. Il sistema di illuminazione ha previsto un funzionamento automatico con fotoperiodo lucebuio pari a 12:12 ore. L'immissione di aria all'interno del sistema è stata effettuata in modalità continua durante l'intera attività sperimentale tramite un compressore, mentre per la $\mathrm{CO}_{2}$ è stata prevista una propagazione dalla durata di sei ore attraverso una bombola di $\mathrm{CO}_{2}$. I due gas sono stati miscelati in un gas samppling tube prima dell'immissione nel mezzo di coltura, garantita da diffusori posti nella parte inferiore della colonna di adsorbimento. La portata di ricircolo è pari a $100 \mathrm{ml} / \mathrm{min}$ e viene immessa da una pompa (LEADFLUID WT600S) che viene azionata per una durata di sei ore e mezzo.

\section{Piani e programma delle attività}

L'attività sperimentale è stata condotta in un arco temporale di 28 giorni, con un programma ope- 
rativo giornaliero di 6 ore durante il fotoperiodo di 12 ore di luce. Le analisi comparative sono state effettuate tra $\mathrm{i}$ due fotobioreattori $\mathrm{PBR}_{1}$ e $\mathrm{PBR}_{2}$ le cui differenze operative sono state indicate in precedenza.

L'avvio del sistema consiste nell'aprire le valvole dei reattori per innescare il circolo del fluido all'interno della colonna di adsorbimento, tramite una pompa. L'aria e la $\mathrm{CO}_{2}$ si miscelano nel gas samppling tube e poi vengono immesse nel sistema attraverso tubazioni collegate nella parte inferiore della colonna di adsorbimento, mentre superiormente viene immessa la portata di ricircolo. Nella colonna di adsorbimento si ottiene una perfetta miscelazione tra frazione liquida e gassosa. Qui si deve tenere sotto controllo il livello del fluido per evitare che si verifichino fuoriuscite e aumenti di pressione; pertanto, quando il livello sale, si apre la valvola della colonna di adsorbimento in modo tale che la miscela fluido-gas entri nel reattore dove le concentrazioni di $\mathrm{CO}_{2}$ vengono assorbite da microalghe della specie Chlorella Vulgaris.

Le analisi sono state condotte sia in fase liquida sia in fase gassosa. Nel primo caso i parametri analizzati sono stati temperatura, $\mathrm{pH}$, ossigeno disciolto, torbidità, assorbanza, clorofilla, solidi sospesi totali e concentrazioni di anioni mentre in fase gassosa sono state monitorate le concentrazioni di $\mathrm{CO}_{2}$ ingresso-uscita e la pressione.

\section{Risultati e discussioni}

Considerando che i due foto-bioreattori hanno previsto stesse condizioni di funzionamento in parallelo, differendo solamente per la lunghezza d'onda dei sistemi di illuminazione, si deduce che risultati eterogeni sono da attribuire esclusivamente alla lunghezza d'onda agente su ogni singolo foto-bioreattore. Per individuare l'efficienza di produzione in termine di biomassa tra i due foto-bioreattori sono state comparate le concentrazioni di solidi sospesi. Con la lunghezza d'onda, emessa dalla sorgente bianca, il foto-bioreattore $\mathrm{PBR}_{1}$ ha raggiunto una concentrazione di biomassa valorizzabile fino a $605 \mathrm{mg} / \mathrm{l}$, inferiore a quanto registrato per il $\mathrm{PBR}_{2}$ in cui la concentrazione di biomassa valorizzabile è stata $825 \mathrm{mg} / \mathrm{l}$ grazie alla lunghezza d'onda emessa dalla luce di colore 
viola. Di conseguenza in $\mathrm{PBR}_{2}$ si sono avuti parametri di performance migliori in termini di tasso di crescita, produttività volumetrica di biomassa e tempo di duplicazione. Anche l'efficienza di rimozione registrata in $\mathrm{PBR}_{2}$ è risultata maggiore ovvero pari a $89 \pm 8 \%$ rispetto a quello con luci bianche dove l'efficienza di rimozione è pari a $83 \pm 9 \%$.

\section{Conclusioni}

I risultati ottenuti durante l'attività hanno dimostrato che il trattamento biologico per le emissioni di gas climalteranti, costituito dal fotobioreattore algale illuminato da luce viola, è risultato più efficiente sia in termini di rimozione di $\mathrm{CO}_{2}$ sia per la produzione di biomassa sfruttabile come materia prima per la conversione in bioprodotti alternativi e rinnovabili, nell'ottica di un processo di decarbonizzazione, sostenibilità ed economia circolare.

\section{References}

1.Tansel, B. Increasing gaps between materials demand and materials recycling rates: A historical perspective for evolution of consumer products and waste quantities. Journal of Environmental Management 276, 111196 (2020).

2.Asselt, H. van. Governing fossil fuel production in the age of climate disruption: Towards an international law of 'leaving it in the ground'. Earth System Governance 9, 100118 (2021).

3.Azam, A., Rafiq, M., Shafique, M. \& Yuan, J. An empirical analysis of the non-linear effects of natural gas nuclear energy, renewable energy and ICT-Trade in leading CO2 emitter countries: Policy towards $\mathrm{CO} 2$ mitigation and economic sustainability. Journal of Environmental Management 286, 112232 (2021).

4.Nisar, A. et al.. Bio-conversion of $\mathrm{CO} 2$ into biofuels and other value-added chemicals via metabolic engineering. Microbiological Research 251, 126813 (2021). 
5.Mukherjee, A., Okolie, J. A., Abdelrasoul, A., Niu, C. \& Dalai, A. K. Review of post-combustion carbon dioxide capture technologies using activated carbon. Journal of Environmental Sciences 83, 46-63 (2019).

6.Arendt, C. A., Hyland, E. G. \& Piliouras, A. The Geological Consequences of Global Climate Change. in Encyclopedia of Geology 510-522 (Elsevier, 2021). doi:10.1016/b978-0-12-4095489.12499-6.

7.Shewchuk, S. R., Mukherjee, A. \& Dalai, A. K. Selective carbon-based adsorbents for carbon dioxide capture from mixed gas streams and catalytic hydrogenation of $\mathrm{CO} 2$ into renewable energy source: A review. Chemical Engineering Science 243, 116735 (2021).

8.Rahman, F. A. et al. Pollution to solution: Capture and sequestration of carbon dioxide (CO 2 ) and its utilization as a renewable energy source for a sustainable future. Renewable and Sustainable Energy Reviews 71, 112-126 (2017).

9.Zhang, S., Liu, L., Zhang, L., Zhuang, Y. \& Du, J. An optimization model for carbon capture utilization and storage supply chain: A case study in Northeastern China. Applied Energy 231, 194-206 (2018).

10.Yadav, S. \& Mondal, S. S. A review on the progress and prospects of oxy-fuel carbon capture and sequestration (CCS) technology. Fuel 308, 122057 (2022).

11.Cuéllar-Franca, R. M. \& Azapagic, A. Carbon capture storage and utilisation technologies: A critical analysis and comparison of their life cycle environmental impacts. Journal of CO2 Utilization 9, 82-102 (2015).

12.Li, L., Zhao, N., Wei, W. \& Sun, Y. A review of research progress on CO2 capture storage, and utilization in Chinese Academy of Sciences. Fuel 108, 112-130 (2013).

13.Daneshvar, E., Wicker, R. J., Show, P.-L. \& Bhatnagar, A. Biologically-mediated carbon capture 
and utilization by microalgae towards sustainable $\mathrm{CO} 2$ biofixation and biomass valorization - $\mathrm{A}$ review. Chemical Engineering Journal 427, 130884 (2022).

14.Vassilev, S. V. \& Vassileva, C. G. Composition properties and challenges of algae biomass for biofuel application: An overview. Fuel 181, 1-33 (2016).

15.Bourgougnon, N., Burlot, A.-S. \& Jacquin, A.-G. Algae for global sustainability? in Advances in Botanical Research 145-212 (Elsevier, 2021). doi:10.1016/bs.abr.2021.01.003.

16.Fernández, F. G. A. et al.. The role of microalgae in the bioeconomy. New Biotechnology 61 , 99-107 (2021).

17.Naddeo, V. \& Taherzadeh, M. J. Biomass valorization and bioenergy in the blue circular economy. Biomass and Bioenergy 149, 106069 (2021).

18.Alami, A. H., Alasad, S., Ali, M. \& Alshamsi, M. Investigating algae for CO2 capture and accumulation and simultaneous production of biomass for biodiesel production. Science of The Total Environment 759, 143529 (2021).

19.Ugwu, C. U., Aoyagi, H. \& Uchiyama, H. Photobioreactors for mass cultivation of algae. Bioresource Technology 99, 4021-4028 (2008).

20.Suparmaniam, U. et al.. Insights into the microalgae cultivation technology and harvesting process for biofuel production: A review. Renewable and Sustainable Energy Reviews 115, 109361 (2019).

21.Yen, H.-W., Hu, I.-C., Chen, C.-Y., Nagarajan, D. \& Chang, J.-S. Design of photobioreactors for algal cultivation. in Biofuels from Algae 225-256 (Elsevier, 2019). doi:10.1016/b978-0-44464192-2.00010-х.

22.Kim, T.-H., Lee, Y., Han, S.-H. \& Hwang, S.-J. The effects of wavelength and wavelength mixing ratios on microalgae growth and nitrogen phosphorus removal using Scenedesmus sp. for 
wastewater treatment. Bioresource Technology 130, 75-80 (2013).

23.Nwoba, E. G., Parlevliet, D. A., Laird, D. W., Alameh, K. \& Moheimani, N. R. Light management technologies for increasing algal photobioreactor efficiency. Algal Research 39, 101433 (2019).

24.Safi, C., Zebib, B., Merah, O., Pontalier, P.-Y. \& Vaca-Garcia, C. Morphology composition, production, processing and applications of Chlorella vulgaris: A review. Renewable and Sustainable Energy Reviews 35, 265-278 (2014).

25.Errami, M. \& Garner, H. A tale of two citations. Nature 451, 397-399 (2008). 
Figure Captions

Figure 1. Sistemi di coltivazione aperti ${ }^{18}$

Figure 2. Sistemi di coltivazione chiusi ${ }^{18}$ 
Figures

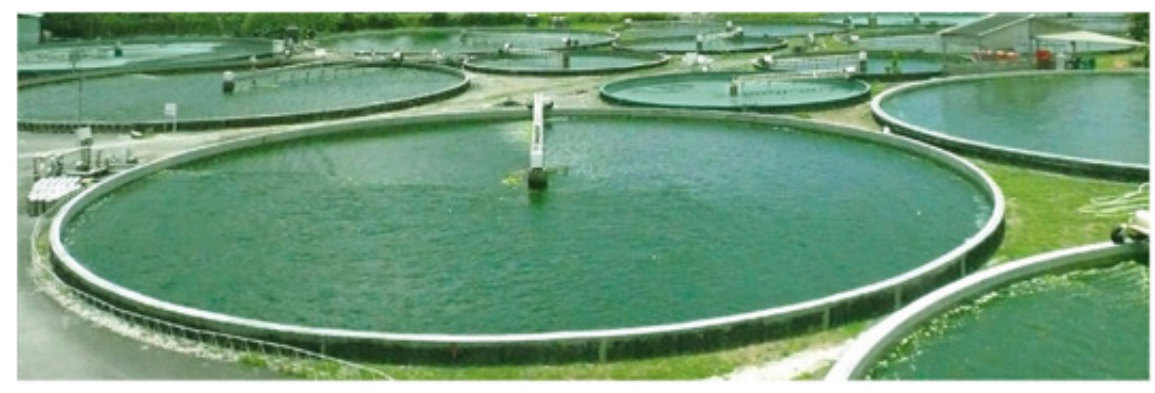

Figure 1: Sistemi di coltivazione aperti ${ }^{18}$ 


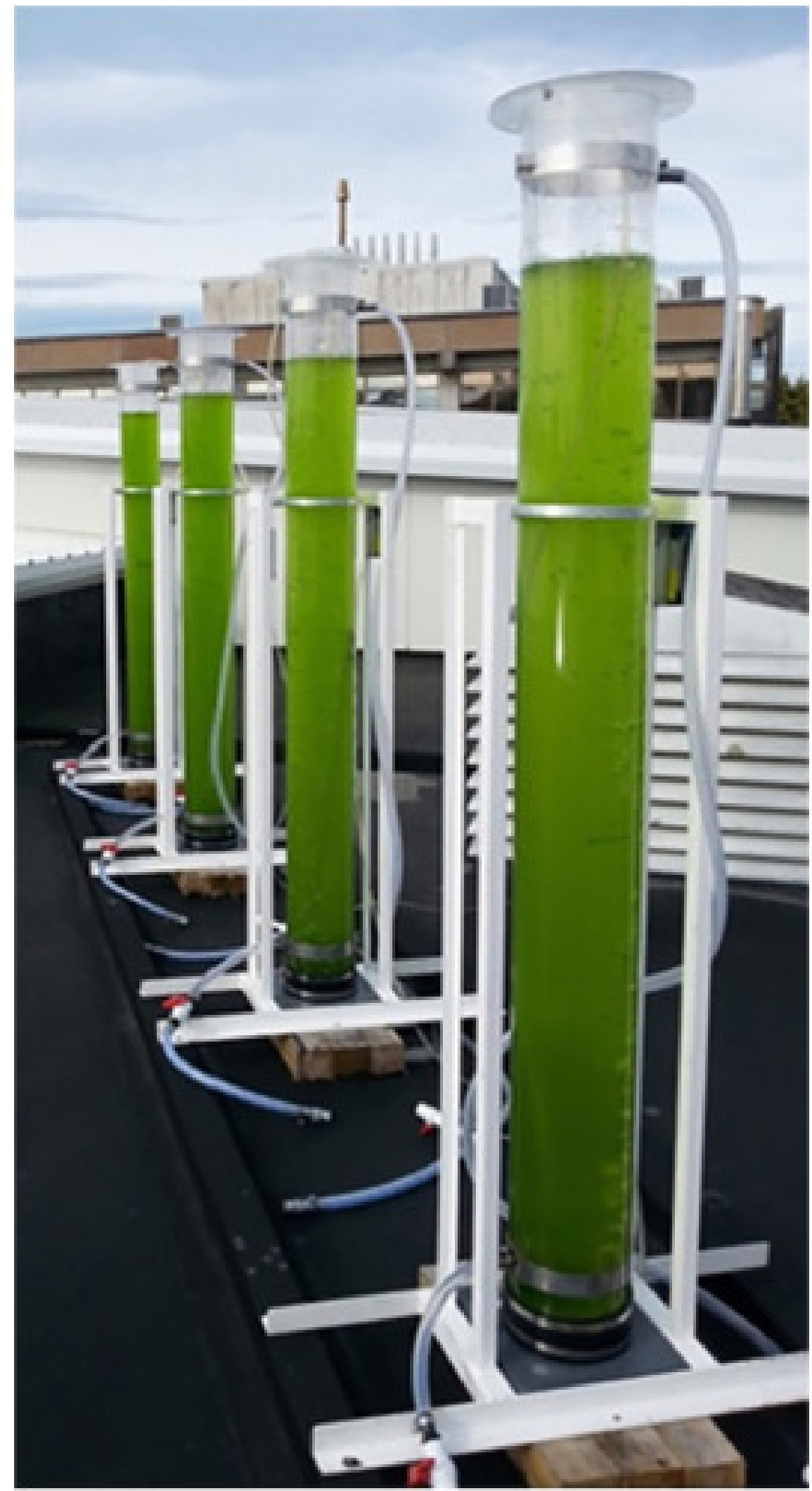

Figure 2: Sistemi di ${ }^{2}$ Coltivazione chiusi ${ }^{18}$ 